\title{
Uso de la Regeneración Natural para la Generación de Plantaciones Productivas de Pino Radiata
}

\author{
Pinilla, J.C. ${ }^{1 *}$; Luengo, K. ${ }^{1}$ y Navarrete, M. ${ }^{2}$. \\ ${ }^{1}$ Ing. Forestal. Instituto Forestal sede Bio Bio, Chile. jpinilla@infor.cl \\ 2 Técnico Forestal. Instituto Forestal, sede Bio Bio. Chile \\ ${ }^{*}$ Autor para correspondencia
}

DOI: https://doi.org/10.52904/0718-4646.2021.472

Recibido: 06.04.21; Aceptado: 25.04.21

\begin{abstract}
RESUMEN
Se presentan antecedentes técnicos y económicos para la toma de decisiones, especialmente de los pequeños y medianos propietarios, respecto a métodos de manejo de la regeneración natural de Pinus radiata, para recuperan las plantaciones afectadas por los mega incendios forestales del año 2017en la región del Biobío. Se analizan dos predios de la comuna de Florida, donde se manejó la regeneración de pino con desbrozadora, herbicidas y herramientas manuales, observándose que el control manual presentó los menores valores de crecimiento en diámetro de cuello, mientras que la utilización del control químico generó los mayores valores en diámetro de cuello y altura.
\end{abstract}

Palabras clave: Pinus radiata, incendios forestales, regeneración natural, manejo forestal.

\begin{abstract}
SUMMARY
Technical and economic antecedents are presented for decision-making, especially for small and medium-sized owners, regarding management methods for the natural regeneration of Pinus radiata, to recover the plantations affected by the mega forest fires of 2017 in the Biobío region.. Two farms in the Florida commune are analyzed, where the regeneration of pine was managed with brush cutters, herbicides and manual tools, observing that the manual control presented the lowest growth values in collar diameter, while the use of chemical control generated the higher values in collar diameter and height.
\end{abstract}

Keywords: Pinus radiata, forest fires, natural regeneration, forest management.

\section{INTRODUCCIÓN}

Los incendios forestales son la principal amenaza que afecta a los bosques en Chile. Las pérdidas económicas y ambientales que generan pueden ser importantes, más aún cuando el rodal está cerca a la edad de cosecha y ha sido manejado para madera clear del mercado de alto valor.

En la temporada 2016-2017 se produjeron grandes incendios que afectaron a más de 500 mil hectáreas y más de 200 mil hectáreas de plantaciones (Raga et al., 2018), además de superficies menores de bosques y matorrales nativos, principalmente entre las Regiones de O'Higgins y Maule (Cuadro 1).

Las superficies quemadas correspondieron mayoritariamente a plantaciones de pino radiata (Pinus radiata D. Don.) y en menor medida de eucaliptos (Eucalyptus globulus Labill.); se concentraron en las regiones del Maule y O'Higgins y afectaron severamente a pequeños y medianos propietarios (Cuadro 2).

La "continuidad y tipo de combustible" es un factor que afecta el comportamiento del incendio, de modo que un adecuado manejo forestal (bosque raleado y especialmente podado) puede reducir su propagación o velocidad de avance y el daño producido en los árboles.

La menor velocidad de avance permite contar con más tiempo para la llegada de las brigadas de combate, menores daños al rodal y menos efecto sobre la rentabilidad esperada de la plantación. 
Cuadro 1. Superficie de Plantaciones Forestales Afectadas por Incendios Forestales por Región

\begin{tabular}{lr}
\hline Región & $\begin{array}{c}\text { Superficie } \\
\text { (ha) }\end{array}$ \\
\hline Coquimbo & 257 \\
\hline Valparaíso & 549 \\
\hline Metropolitana & 295 \\
\hline O’Higgins & 34.427 \\
\hline Maule & 128.156 \\
\hline Biobío & 39.246 \\
\hline Araucanía & 1.778 \\
\hline Total & $\mathbf{2 0 4 . 7 0 8}$ \\
\hline (Fuente: Raga et al., 2018) &
\end{tabular}

Cuadro 2. Superficies de Plantaciones Quemadas por Región y Especie en Poder de Pequeños y Medianos Propietarios

\begin{tabular}{|c|c|c|c|c|c|c|c|c|c|}
\hline \multirow[b]{2}{*}{ Región } & \multicolumn{4}{|c|}{ Medianos Propietarios } & \multicolumn{4}{|c|}{ Pequeños Propietarios } & \multirow{2}{*}{ Total } \\
\hline & Pino & Eucalipto & Otras & Total & $\begin{array}{r}\text { Pino } \\
\text { (ha) }\end{array}$ & Eucalipto & Otras & Total & \\
\hline Coquimbo & & & & & & & 257 & 257 & 257 \\
\hline Valparaíso & & 32 & & 32 & 22 & 469 & & 491 & 523 \\
\hline Metropolitana & & 260 & & 260 & & 35 & 25 & 60 & 320 \\
\hline O’Higgins & 11.172 & 3.932 & 13 & 15.117 & 4.827 & 6.733 & 4 & 11.564 & 26.681 \\
\hline Maule & 14.493 & 1.219 & 13 & 15.725 & 26.697 & 5.797 & 3 & 32.497 & 48.222 \\
\hline Biobío & 845 & 1.521 & & 2.366 & 5.174 & 8.601 & 35 & 13.810 & 16.176 \\
\hline Araucanía & 111 & 11 & & 122 & 301 & 296 & 24 & 621 & 743 \\
\hline Total & 26.621 & 6.975 & 26 & 33.622 & 37.021 & 21.931 & 348 & 59.300 & 92.922 \\
\hline
\end{tabular}

(Fuente: Raga et al., 2018)

El manejo forestal también es una herramienta para enfrentar situaciones post incendio y facilitar el establecimiento de un nuevo bosque en un menor tiempo y costo. Contar con información para abordar escenarios post incendios y sobre todo conocer opciones para realizar la gestión forestal, son elementos claves para recuperar una superficie quemada.

La necesidad de recuperar las plantaciones que fueron afectadas por los grandes incendios forestales que afectaron al país en 2017 ha motivado la búsqueda de opciones para la rápida recuperación productiva de estos bosques. Entre ellas, el uso de la regeneración natural se visualiza como una alternativa interesante, pues en el caso de pino radiata, se produce profusamente tras el efecto del fuego sobre los conos que contienen las semillas. El calor provoca la apertura de los conos y dispersión de las semillas, incentivando también su germinación.

En efecto, tras los incendios pino radiata puede generar verdaderas "alfombras" de regeneración, con un alto número de plantas por hectárea, que brindan un escenario donde el manejo de la regeneración natural puede ser considerada como opción silvícola (Buesa, 2003; Castelán y Arteaga, 2009).

Esta situación permitiría el restablecimiento de cubierta arbórea sobre la superficie quemada, constituyendo una opción que puede ser implementada por pequeños y medianos propietarios, segmentos que normalmente no tienen los recursos para establecer plantaciones, y menos aún bajo la actual situación que los encuentra descapitalizados (Raga et al., 2018).

La experiencia extranjera indica que es posible usar la regeneración natural de pino radiata que se produce tras un incendio forestal. En Nueva Zelandia, Australia y México, entre otros, se han usado diversos tratamientos o modelos de manejo con este fin. En general, la decisión de utilizar o no la regeneración se debe basar en consideraciones sobre recursos disponibles, edad y estado de la regeneración (calidad, forma, altura, densidad, espaciamiento final deseado y otras), y la topografía, entre otras. A medida que la regeneración tiene mayor edad, se dificulta el manejo de la misma.

Por lo anterior, en este trabajo se plantea un estudio para generar antecedentes técnicos y económicos para la toma de decisiones respecto al uso de la regeneración natural de pino radiata como herramienta 
para la recuperación productiva de plantaciones quemadas, particularmente de pequeños y medianos propietarios forestales de las regiones de Ñuble y Bio Bio.

El estudio plantea caracterizar situaciones con presencia de regeneración de pino radiata producto de los incendios forestales del año 2017, establecer métodos a aplicar para su manejo, aplicar estos manejos y evaluar los resultados desde el punto de vista técnico y financiero. En este sentido, el objetivo es generar antecedentes técnicos que permitan la toma de decisiones para la utilización de la regeneración natural de pino radiata como herramienta de gestión para la restauración productiva de bosques.

\section{MATERIAL Y MÉTODO}

Se generó cobertura cartográfica de la intensidad del daño en las plantaciones afectadas por los grandes incendios del año 2017 en las regiones de Ñuble y Bio Bio. A partir de ella se identificó a las comunas con mayor daño y se obtuvo información de las superficies de plantaciones forestales (pino y eucalipto) afectadas por región y tipo de propietario (Cuadro 3). Una vez identificadas las comunas de interés, se seleccionó los sitios donde se realizaría el estudio, usando la información entregada por el Programa de Actualización de Plantaciones de INFOR.

Cuadro 3. Plantaciones de Pino Radiata Afectadas por Incendios 2017 según Región

\begin{tabular}{|c|c|c|c|c|c|}
\hline \multirow{2}{*}{ Región } & \multicolumn{4}{|c|}{ Tipo de Propietario } & \multirow{2}{*}{$\begin{array}{l}\text { Total } \\
\text { (ha) }\end{array}$} \\
\hline & GE & EM & MP & PP & \\
\hline O'Higgins & 5.901 & 2.392 & 11.172 & 4.817 & 23.472 \\
\hline Maule & 75.784 & 2.232 & 14.493 & 26.697 & 119.206 \\
\hline Bio Bio & 12.861 & 782 & 845 & 5.174 & 19.663 \\
\hline Total & 93.736 & 5.406 & 26.510 & 36.688 & 162.340 \\
\hline (\%) & 58 & 3 & 16 & 23 & 100 \\
\hline
\end{tabular}

\begin{tabular}{lrrrrrr}
\hline \multirow{1}{*}{ Región } & \multicolumn{5}{c}{$\begin{array}{c}\text { Rango de Edad } \\
\text { (años) }\end{array}$} & $\begin{array}{l}\text { Total } \\
\text { (ha) }\end{array}$ \\
\cline { 2 - 7 } & \multicolumn{1}{c}{$\mathbf{0 - 5}$} & \multicolumn{1}{c}{$\mathbf{6 - 1 3}$} & $\mathbf{1 4 - 1 7}$ & $\mathbf{1 8 - 2 3}$ & \multicolumn{1}{c}{$>$ 23 } & \\
\hline O'Higgins & 2.687 & 7.414 & 4.350 & 6.304 & 2.717 & 23.472 \\
\hline Maule & 19.709 & 41.621 & 19.835 & 24.861 & 13.180 & 119.206 \\
\hline Bio Bio & 2.420 & 5.997 & 4.435 & 4.354 & 2.456 & 19.663 \\
\hline Total & $\mathbf{2 4 . 8 1 6}$ & $\mathbf{5 5 . 0 3 2}$ & $\mathbf{2 8 . 6 1 9}$ & $\mathbf{3 5 . 5 1 9}$ & $\mathbf{1 8 . 3 5 4}$ & $\mathbf{1 6 2 . 3 4 0}$ \\
\hline
\end{tabular}

(Fuente: INFOR, 2018)

GE: Gran empresa; EM: Empresa mediana; MP: Mediano propietario;

PP: Pequeño propietario

Cuadro 4. Plantaciones de Eucalipto Afectadas por Incendios 2017 según Región

\begin{tabular}{|c|c|c|c|c|c|}
\hline \multirow{2}{*}{ Región } & \multicolumn{4}{|c|}{ Tipo de Propietario } & \multirow{2}{*}{$\begin{array}{l}\text { Total } \\
\text { (hHa) }\end{array}$} \\
\hline & GE & EM & MP & PP & \\
\hline O'Higgins & 31 & 232 & 3.921 & 6.733 & 10.918 \\
\hline Maule & 1.387 & 218 & 1.219 & 5.797 & 8.621 \\
\hline Biobío & 8.029 & 664 & 1.521 & 8.531 & 18.746 \\
\hline Total & 9.447 & 1.115 & 6.692 & 21.061 & 38.284 \\
\hline$(\%)$ & 25 & 3 & 17 & 55 & 100 \\
\hline
\end{tabular}

\begin{tabular}{lcccr}
\hline \multicolumn{1}{c}{ Región } & \multicolumn{3}{c}{$\begin{array}{c}\text { Rango de Edad } \\
\text { (años) }\end{array}$} & \multicolumn{1}{l}{$\begin{array}{l}\text { Total } \\
\text { (ha) }\end{array}$} \\
\cline { 2 - 4 } & $\mathbf{0 - 6}$ & $\mathbf{7 - 1 2}$ & $\mathbf{> 1 2}$ & \\
\hline O'Higgins & 2.244 & 4.976 & 3.697 & 10.918 \\
\hline Maule & 1.886 & 3.474 & 3.261 & 8.621 \\
\hline Biobío & 5.747 & 6.696 & 6.303 & 18.746 \\
\hline Total & 9.876 & 15.146 & 13.262 & 38.284 \\
\hline
\end{tabular}

(Fuente: INFOR, 2018)

GE: Gran empresa; EM: Empresa mediana; MP: Mediano propietario; PP: Pequeño propietario 


\section{Situaciones Evaluadas}

En la primera etapa del estudio se seleccionó los predios Lo Carmen y Santa Elisa, en la comuna de Florida, sector Copiulemu (Figura 1 y 2, y Cuadro 5) los que cumplían con los siguientes requisitos necesarios para el estudio:

Presencia de plantaciones de pino radiata afectadas por el incendio del año 2017, con regeneración natural de la especie.

Pertenecer a pequeños o medianos propietarios.

Ubicados en sectores representativos de las situaciones y suelos de la comuna.

Propietarios dispuestos a la realización de las actividades programadas en el estudio.

Propietarios que manifestaran su intención de continuar con la actividad forestal.

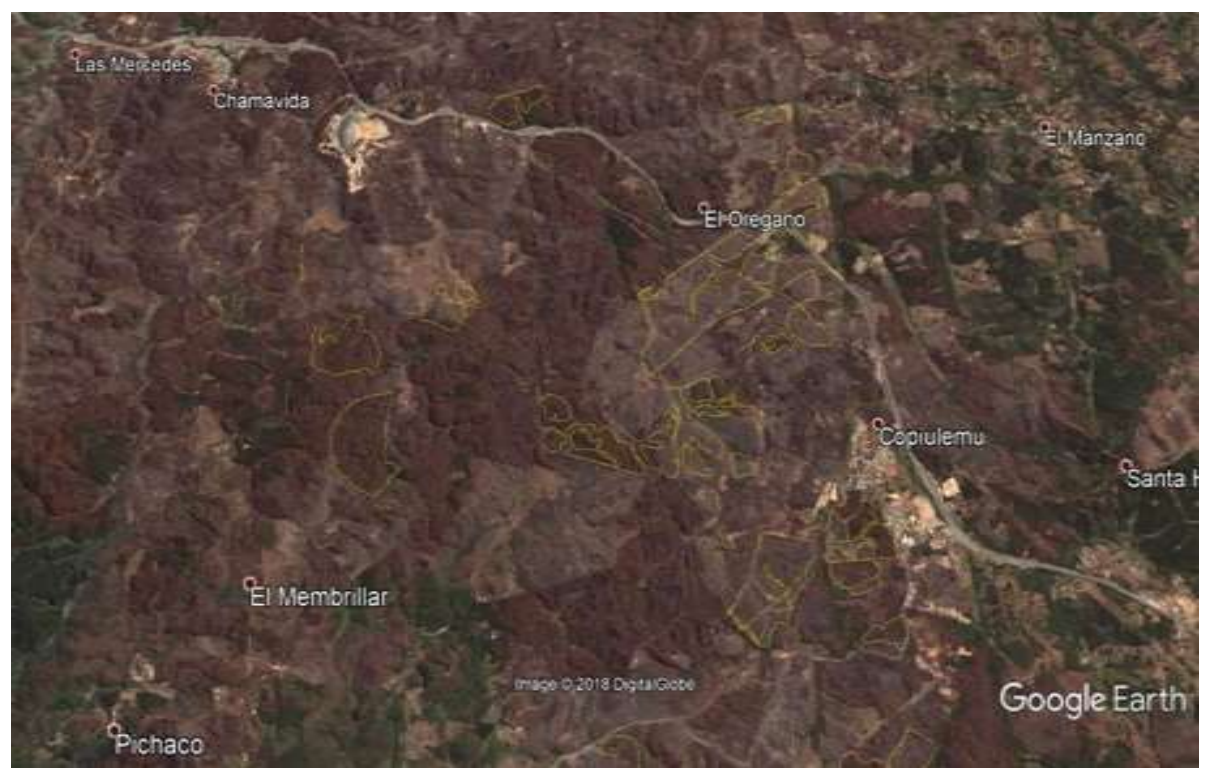

Figura 1. Situación Rodales Afectados Por Incendios Forestales, Sector Copiulemu, Comuna de Florida, Región del Bio Bio

Cuadro 5. Predios Seleccionados para Realización Estudio

\begin{tabular}{lll}
\hline Predio & Coordenadas & Especie Afectada \\
\hline Lo Carmen & $18 \mathrm{H} 693623 ; 5912742$ & Pino y eucalipto \\
\hline Santa Elisa & $18 \mathrm{H} 694188 ; 5915342$ & Pino \\
\hline
\end{tabular}

En el predio Lo Carmen se instaló parcelas de estudio en una superficie homogénea de pino cosechado, con abundante regeneración natural, que en algunas zonas se veía disminuida por la acumulación de desechos de la explotación (Figura 3).

En el predio Santa Elisa la regeneración de pino se presentaba en dos situaciones distintas:

(i) una con una alta presencia de retamilla, que influyó en la cantidad de plantas regeneradas.

(ii) otra con una proliferación masiva de regeneración, de menor crecimiento que la anterior, pero sin presencia de especies competidoras (Figura 4). En ambas situaciones se estableció parcelas de estudio. 

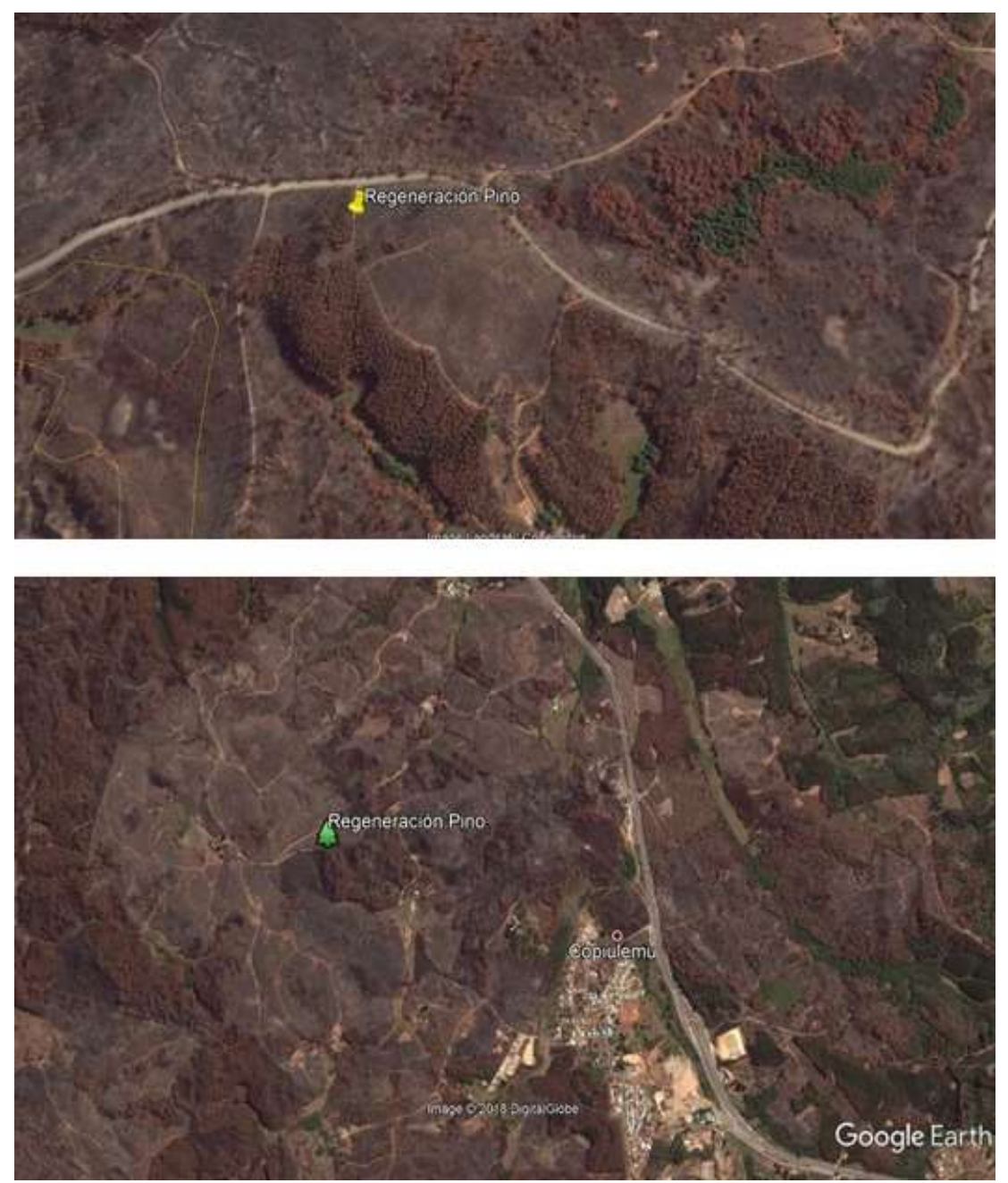

Figura 2. Situación Febrero de 2017 Predio Lo Carmen (arriba) y Santa Elisa (abajo)

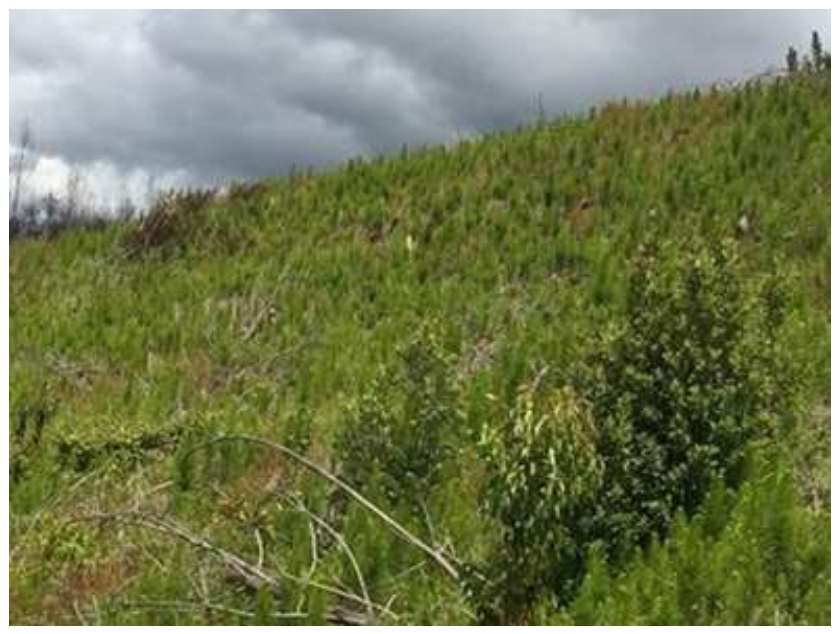

Figura 3. Vista Predio Lo Carmen 


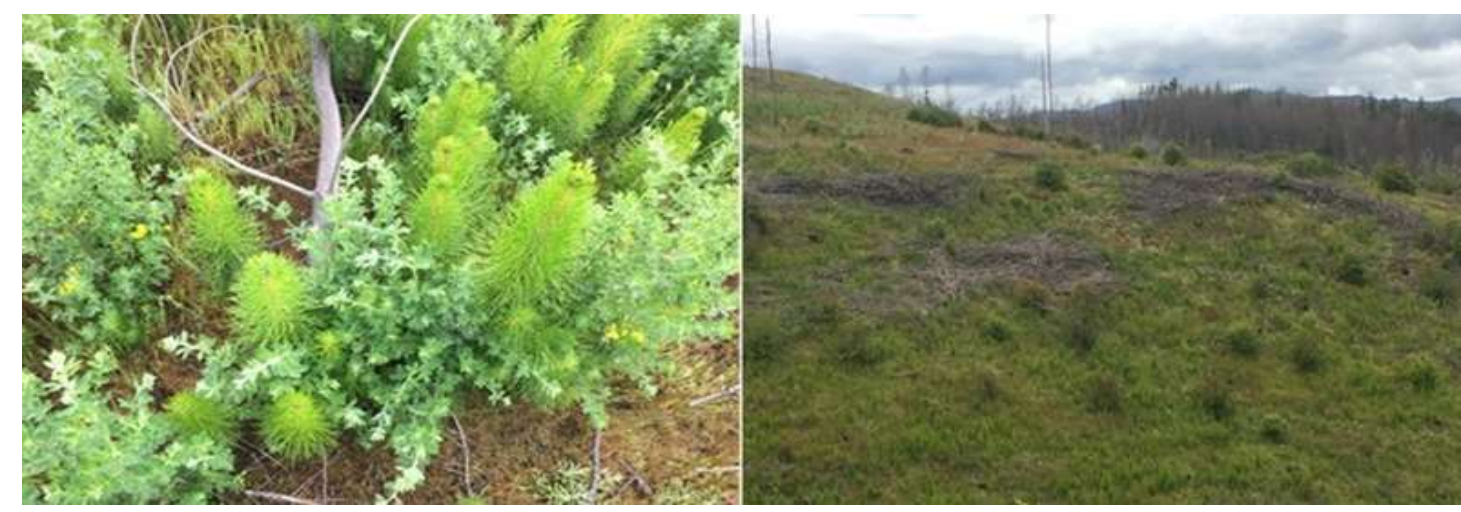

Figura 4. Vistas Predio Santa Elisa

\section{Tratamientos de Manejo Evaluados e Instalación de Parcelas}

Se evaluó tres métodos para el manejo de la regeneración de pino:

\section{Desbrozadora}

Control químico

Control manual con rozón, machete y extracción de las plantas

En predio Lo Carmen se instaló una parcela de $1.000 \mathrm{~m}^{2}$ (Parcela Lo Carmen) y en predio Santa Elisa dos, una en cada situación descrita y denominadas "Parcela Santa Isabel ladera" y "Parcela Santa Isabel cima" (Figuras 5 y 6). Los métodos aplicados a cada parcela se muestran en el Cuadro 6. Fotografías de la aplicación de los métodos evaluados se presentan en las Figuras 7 a 9 . El aspecto final de las parcelas manejadas con cada uno de los métodos evaluados se presenta en las Figuras 10 a 12. Durante el manejo de la regeneración se realizó estudio de tiempo rendimiento de la faena.

Cuadro 6. Métodos de Manejo de Regeneración Natural de Pino Aplicados a las Parcelas Evaluadas

\begin{tabular}{ll}
\hline Sector & Tipo de Manejo \\
\hline \multirow{2}{*}{ Parcela "Lo Carmen" } & Desbrozadora \\
\cline { 2 - 2 } & Manual \\
\cline { 2 - 2 } Parmico \\
\hline Parcela "Santa Isabel" Ladera & Químico \\
\cline { 2 - 2 } & Desbrozadora \\
\hline
\end{tabular}
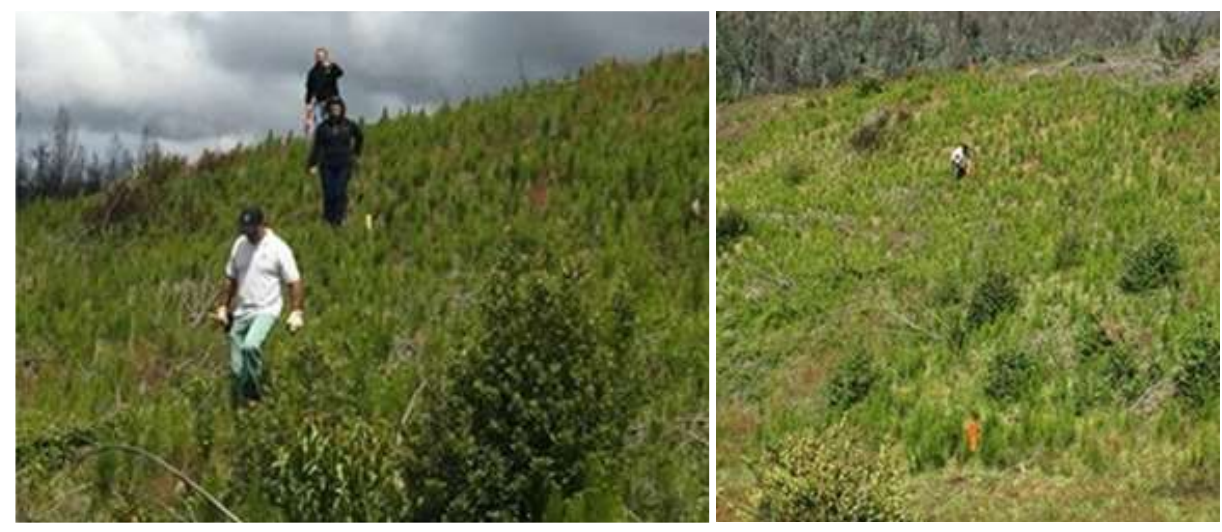

Figura 5. Instalación Parcela Predio Lo Carmen 

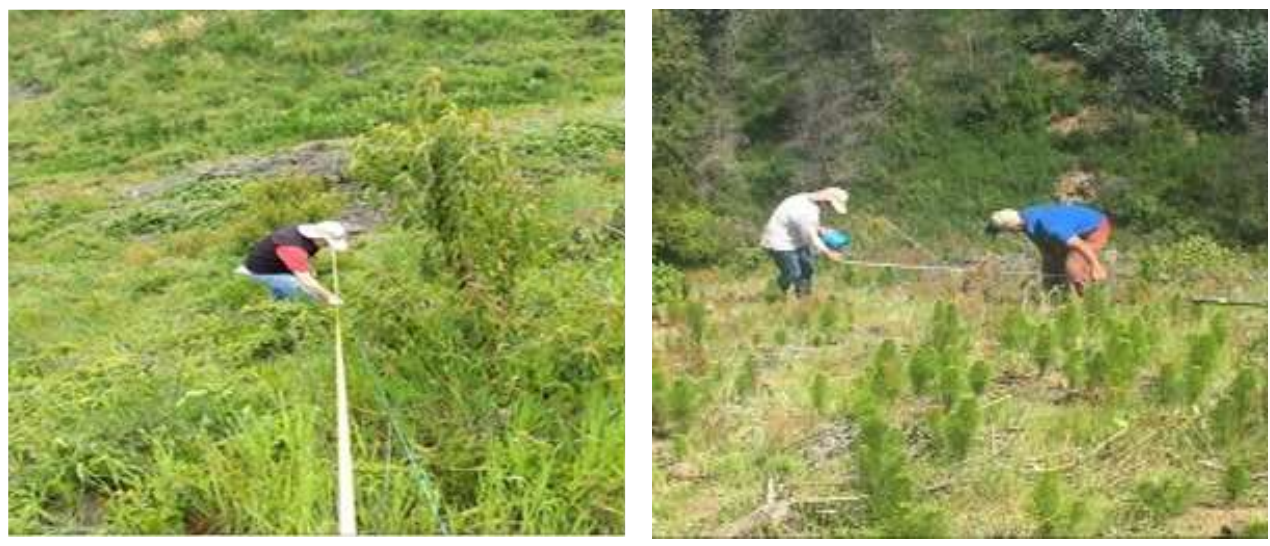

Figura 6. Instalación Parcela Predio Santa Elisa
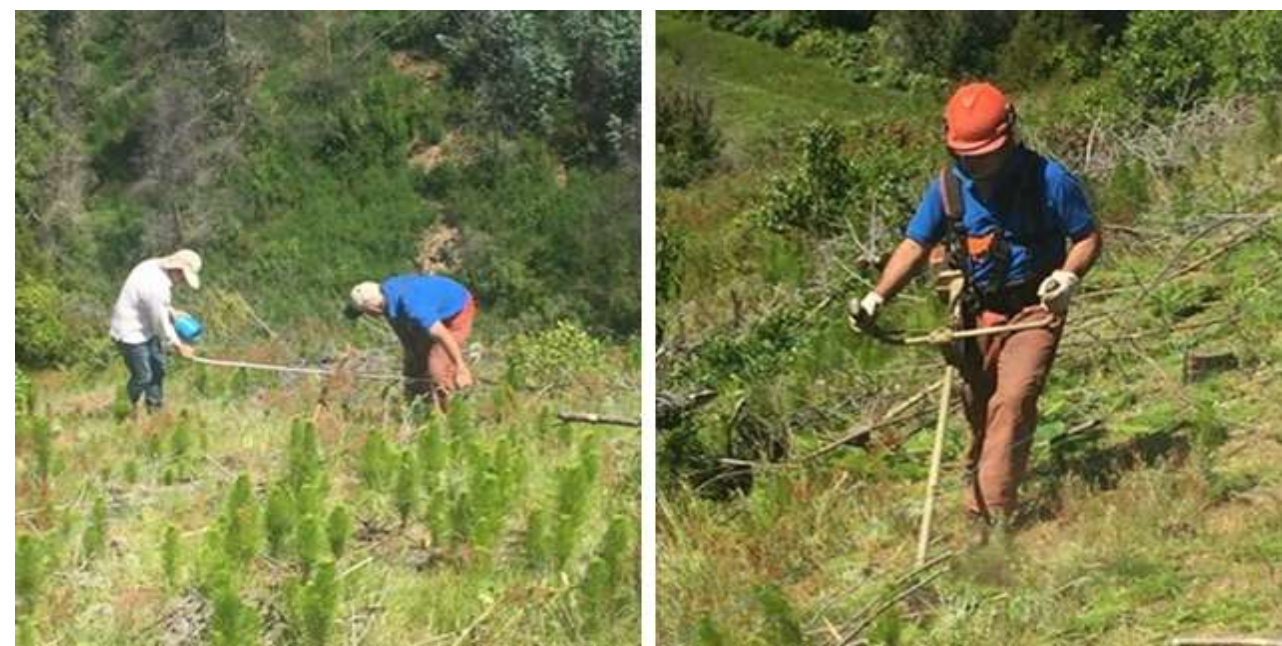

Figura 7. Preparación de los Sectores a Intervenir y Aplicación Método de Manejo Utilizando Desbrozadora
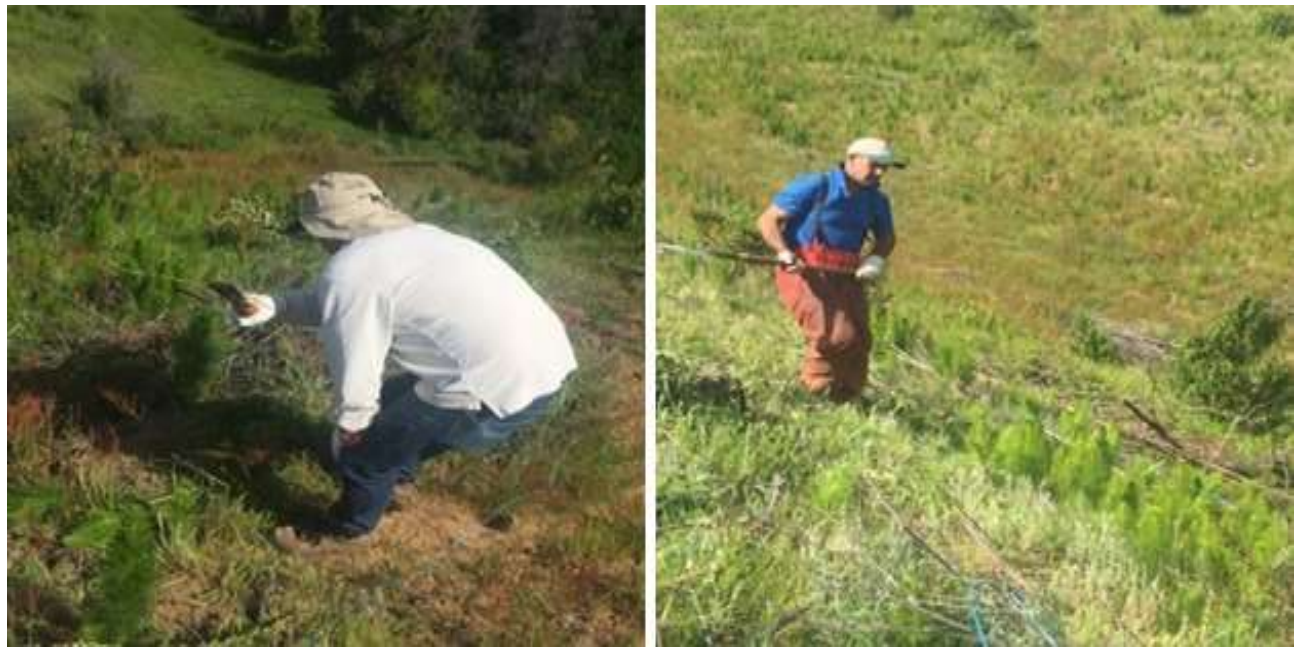

Figura 8. Aplicación Método de Manejo Manual 


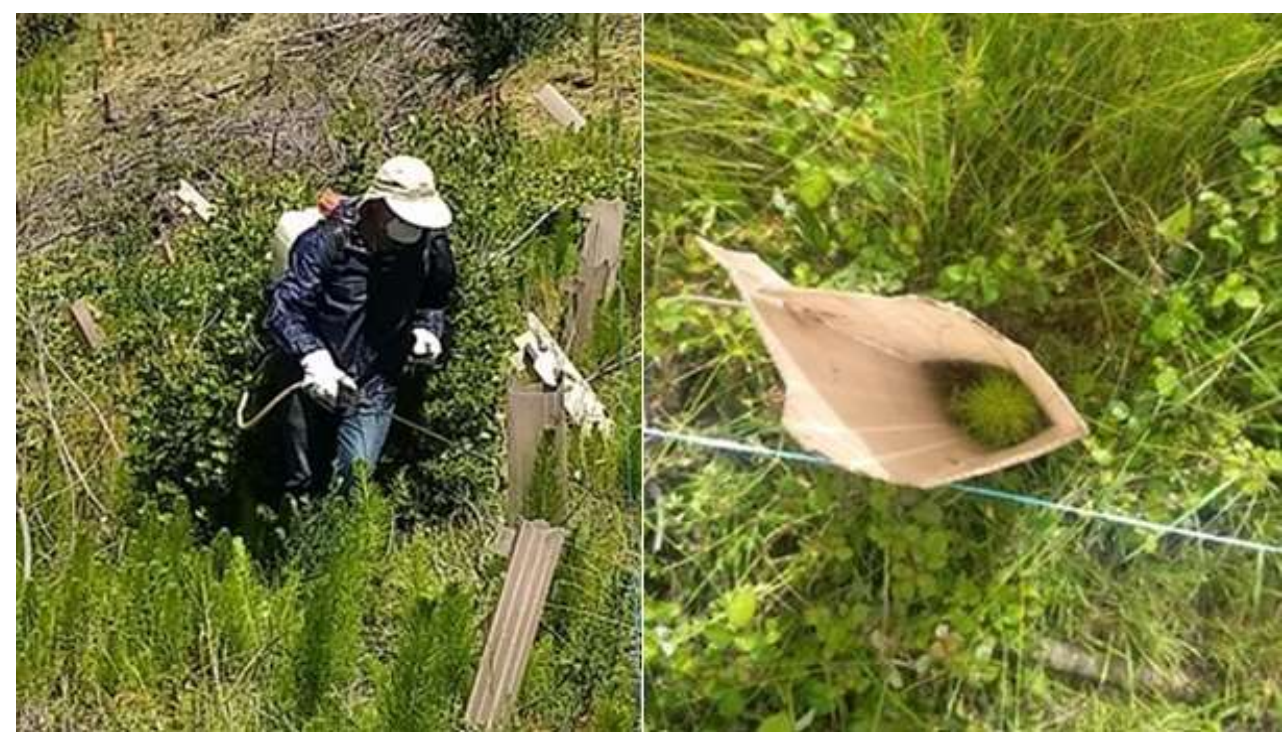

Figura 9. Aplicación Método de Manejo Mediante Control con Productos Químicos, se Observa Detalle de Protección de las Plantas Utilizado

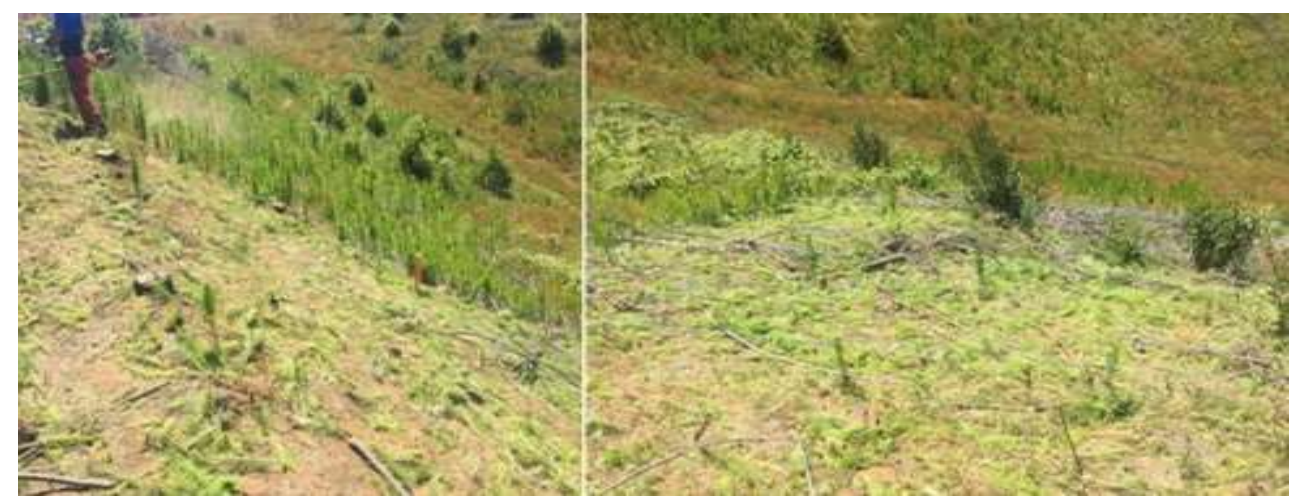

Figura 10. Situación Final luego de Manejo Manual
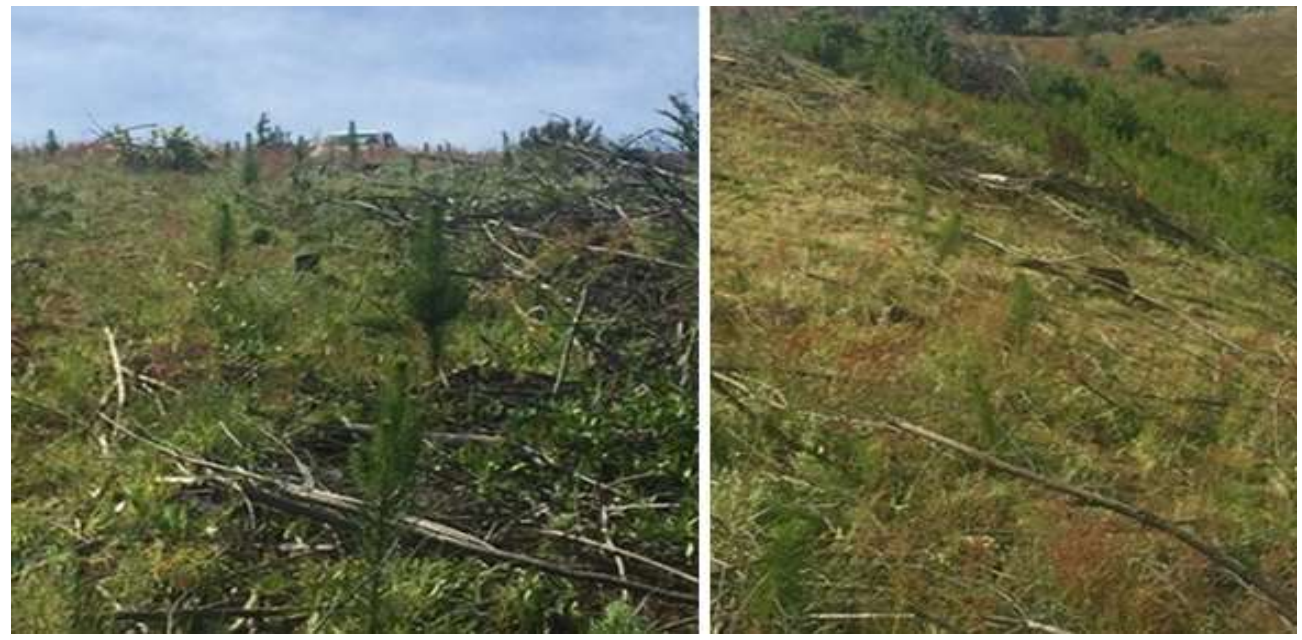

Figura 11. Situación Final luego de Manejo con Desbrozadora 

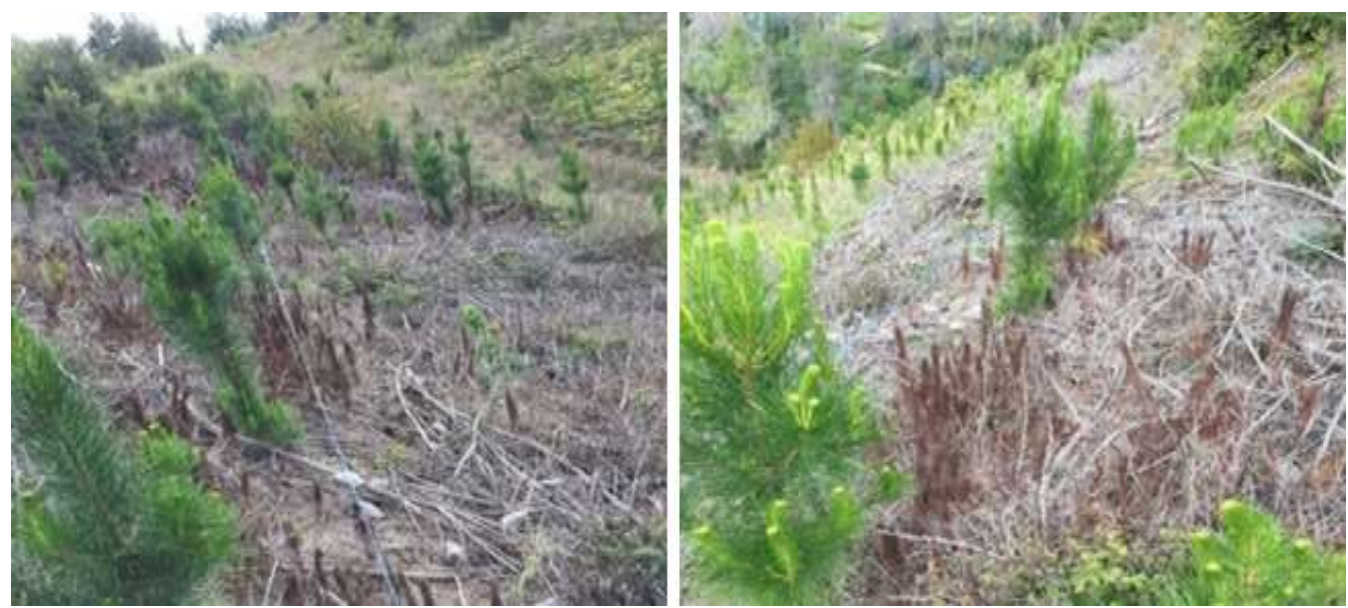

Figura 12. Situación Final luego de Manejo Químico

Previo a aplicar los métodos de manejo se estimó la cantidad de plantas de regeneración y la altura de las mismas. Para ello en cada parcela de $1.000 \mathrm{~m}^{2}$ se instaló un mínimo de 3 parcelas cuadradas de $1 \mathrm{~m}^{2}$ cada una y. en caso de existir una cantidad diferente de plantas por parcela superior a una desviación estándar, se agregaban 2 parcelas más (Figuras 13 y 14). Esta situación se verificó en la primera parcela instalada en el predio Santa Elisa (Pinilla et al., 2019).
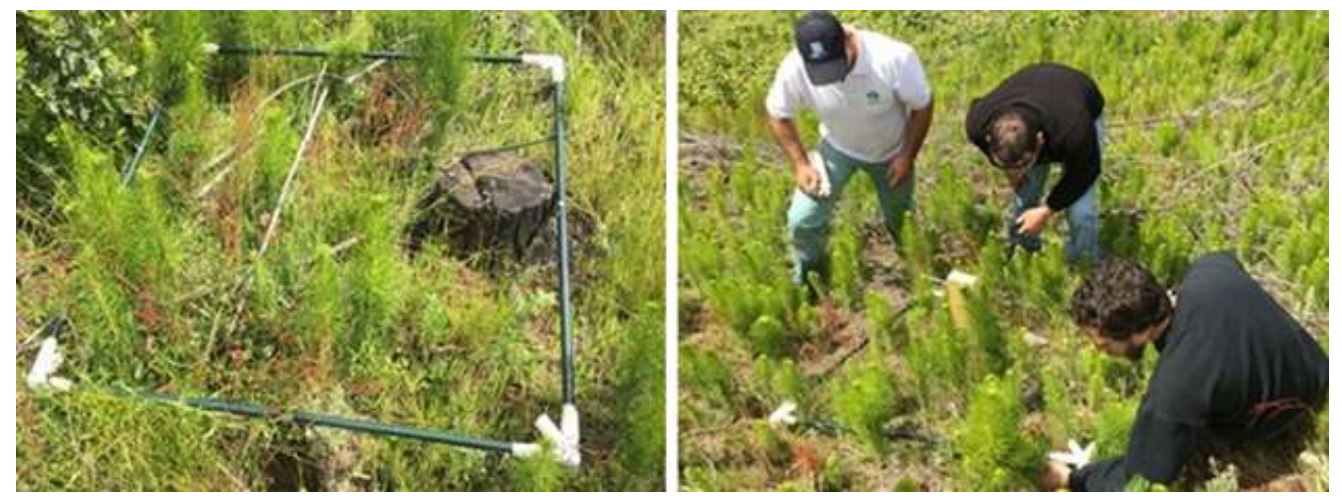

Figura 13. Instalación Parcelas para Estimación de la Regeneración y Conteo de Plantas de Pino

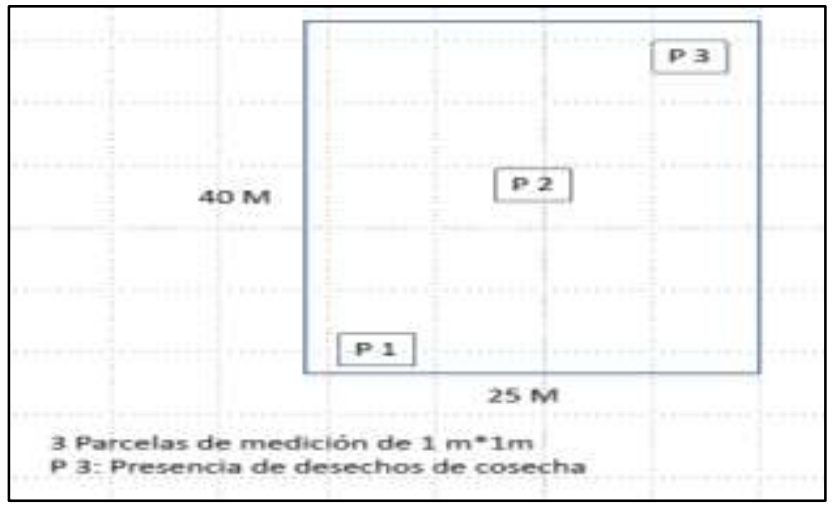

Figura 14. Diseño Parcela Experimental 


\section{RESULTADOS Y DISCUSIÓN}

\section{Situación Inicial y Rendimiento de Faenas de Manejo}

De acuerdo con las mediciones realizadas durante el año 2018, previo a aplicar los tratamientos de manejo, la regeneración de pino radiata presentaba una densidad promedio de 310.000 plantas por hectárea, equivalente a un distanciamiento aproximado de $18 \mathrm{~cm}$, y una altura media de $30,8 \mathrm{~cm}$ (Cuadro 7).

Cuadro 7. Cantidad de Plantas de Regeneración Natural de Pino Radiata

\begin{tabular}{|c|c|c|c|c|c|c|c|c|}
\hline \multirow{3}{*}{ Predio } & \multicolumn{6}{|c|}{$\begin{array}{l}\text { Plantas por Parcela Regeneración } \\
\left(\mathbf{N}^{\circ}\right)\end{array}$} & \multirow{3}{*}{$\begin{array}{l}\text { Densidad } \\
\text { (pl/ha) }\end{array}$} & \multirow{3}{*}{$\begin{array}{l}\text { Altura } \\
(\mathbf{c m})\end{array}$} \\
\hline & \multicolumn{5}{|c|}{ Parcela } & \multirow[t]{2}{*}{ Promedio } & & \\
\hline & 1 & 2 & 3 & 4 & 5 & & & \\
\hline Lo Carmen & 38 & 53 & 9 & & & 33 & 330.000 & 32,2 \\
\hline Santa Elisa I & 13 & 38 & 10 & 13 & 12 & 17 & 170.000 & 33,5 \\
\hline Santa Elisa II & 53 & 42 & 33 & & & 43 & 430.000 & 26,8 \\
\hline Promedio & & & & & & 31 & 310.000 & 30,8 \\
\hline
\end{tabular}

Los resultados de la evaluación de tiempo y rendimiento se resumen en el Cuadro 8.

Cuadro 8. Resultados Obtenidos de la Aplicación de los Manejos según Sector

\begin{tabular}{|c|c|c|c|c|c|c|}
\hline Sector & Tipo de Manejo & $\begin{array}{c}\text { Instalación de } \\
\text { Línea }\end{array}$ & $\begin{array}{c}\text { Selección de } \\
\text { Planta }\end{array}$ & $\begin{array}{c}\text { Tiempo de } \\
\text { Manejo }\end{array}$ & $\begin{array}{c}\text { Insumo para } \\
500 \mathrm{~m}^{2}\end{array}$ & $\begin{array}{c}\text { Número de } \\
\text { Personas }\end{array}$ \\
\hline \multirow{3}{*}{$\begin{array}{l}\text { Parcela "Lo } \\
\text { Carmen" }\end{array}$} & Desbrozadora & $32 \min 14 \mathrm{~s}$ & $39 \min 52 \mathrm{~s}$ & $\begin{array}{c}1 \text { hora } 54 \mathrm{~min} \\
30 \mathrm{~s}\end{array}$ & $\begin{array}{c}1,5 \text { estaque } \\
(+)\end{array}$ & 2 \\
\hline & Manual & $18 \min 33 \mathrm{~s}$ & \multicolumn{2}{|c|}{1 hora $18 \mathrm{~min} 11 \mathrm{~s}$} & 0 & 3 \\
\hline & Químico & & $55 \min 36 \mathrm{~s}$ & $45 \min 44 \mathrm{~s}$ & $\begin{array}{c}3,5 \text { estaque } \\
(++)\end{array}$ & 2 \\
\hline \multirow{2}{*}{$\begin{array}{l}\text { Parcela "Santa } \\
\text { Isabel" - Ladera }\end{array}$} & Químico & $29 \min 45 \mathrm{~s}$ & $\begin{array}{c}1 \text { hora } 04 \mathrm{~min} \\
34 \mathrm{~s}\end{array}$ & $28 \mathrm{~min} 31 \mathrm{seg}$ & $\begin{array}{c}4 \text { estanques } \\
(++)\end{array}$ & 2 \\
\hline & Desbrozadora & & $\begin{array}{c}1 \text { hora } 26 \mathrm{~min} \\
46 \mathrm{seg}\end{array}$ & $\begin{array}{c}2 \text { hora } 42 \mathrm{~min} \\
04 \mathrm{seg}\end{array}$ & 3 & 2 \\
\hline $\begin{array}{l}\text { Parcela "Santa } \\
\text { Isabel" - Cima }\end{array}$ & Desbrozadora (-) & $13 \min 15 \mathrm{~s}$ & $\begin{array}{c}1 \text { hora } 26 \mathrm{~min} \\
30 \mathrm{~s}\end{array}$ & $\begin{array}{c}2 \text { hora } 54 \mathrm{~min} \\
23 \mathrm{~s}\end{array}$ & $\begin{array}{c}6 \text { estanques } \\
(++)\end{array}$ & 2 \\
\hline
\end{tabular}

(+) Mezcla de aceite con bencina

(++) Mezcla químicos con agua (proporción cada 15 litros de agua 300 cc de químicos) capacidad del estanque 15 L

(-) El manejo se hizo a los $1.000 \mathrm{~m}^{2}$, a diferencia de las otras que cada manejo se realizó en $500 \mathrm{~m}^{2}$

La densidad inicial de la regeneración influye en el rendimiento de los métodos de manejo posibles de aplicar.

En el caso del uso de desbrozadora, el efecto de la densidad sobre el rendimiento de la faena, en jornadas por hectárea, se puede apreciar en los resultados del Cuadro 9.

Cuadro 9. Rendimiento de Faena de Manejo de Regeneración Natural con Desbrozadora en Situaciones con Diferentes Densidades de Plantas

\begin{tabular}{llcc}
\hline \multicolumn{1}{c}{ Sector } & Método & $\begin{array}{c}\text { Densidad } \\
(\mathrm{pl} / \mathrm{ha})\end{array}$ & Jornadas/ha \\
\hline Parcela "Lo Carmen" & Desbrozadora & 172.000 & 11 \\
\hline Parcela "Santa Isabel" - Ladera & Desbrozadora & 285.000 & 13 \\
\hline Parcela "Santa Isabel" - Cima & Desbrozadora $(-)$ & 426.667 & 21 \\
\hline
\end{tabular}

(-) El manejo se hizo a los $1000 \mathrm{~m}^{2}$, a diferencia de las otras que cada manejo se realizó en $500 \mathrm{~m}^{2}$ 
El tamaño de las plantas de la regeneración también influye sobre la eficiencia del manejo a aplicar. A mayor tamaño de plantas se dificulta la aplicación de todos los métodos evaluados. Por lo mismo, el manejo de la regeneración debe iniciarse tempranamente.

\section{Evaluación de Unidades}

El monitoreo realizado indica que las unidades presentan un adecuado desarrollo y un claro efecto de los métodos de manejo utilizados. Se destaca el efecto del control químico, el que registra los mejores crecimientos en la regeneración manejada y se observa una nueva regeneración de semillas con presencia de plantas de tamaño menor, lo que deberá ser también monitoreado.

\section{- Evaluación Unidad Lo Carmen}

Los valores medios de altura y diámetro de cuello de las plantas de regeneración sometidas a los distintos métodos de intervención evaluados se resumen en el Cuadro 10.

Cuadro 10. Situación de Plantas de Regeneración Manejadas en Unidad Lo Carmen

\begin{tabular}{ccccccc}
\hline & \multicolumn{6}{c}{ Tratamiento de Manejo } \\
\cline { 2 - 7 } Año & \multicolumn{2}{c}{ Manual } & \multicolumn{2}{c}{ Desbrozadora } & \multicolumn{2}{c}{ Químico } \\
\cline { 2 - 7 } & $\begin{array}{c}\text { DC } \\
(\mathbf{m m})\end{array}$ & $\begin{array}{c}\text { Altura } \\
(\mathbf{c m})\end{array}$ & $\begin{array}{c}\text { DC } \\
(\mathbf{m m})\end{array}$ & $\begin{array}{c}\text { Altura } \\
(\mathbf{c m})\end{array}$ & $\begin{array}{c}\text { DC } \\
(\mathbf{m m})\end{array}$ & $\begin{array}{c}\text { Altura } \\
(\mathbf{c m})\end{array}$ \\
\hline $\mathbf{2 0 1 9}$ & 17,7 & 91,3 & 18,6 & 91,3 & 23,4 & 120,7 \\
\hline $\mathbf{2 0 2 0}$ & 24,5 & 128,1 & 21,6 & 124,3 & 34,6 & 201,0 \\
\hline
\end{tabular}
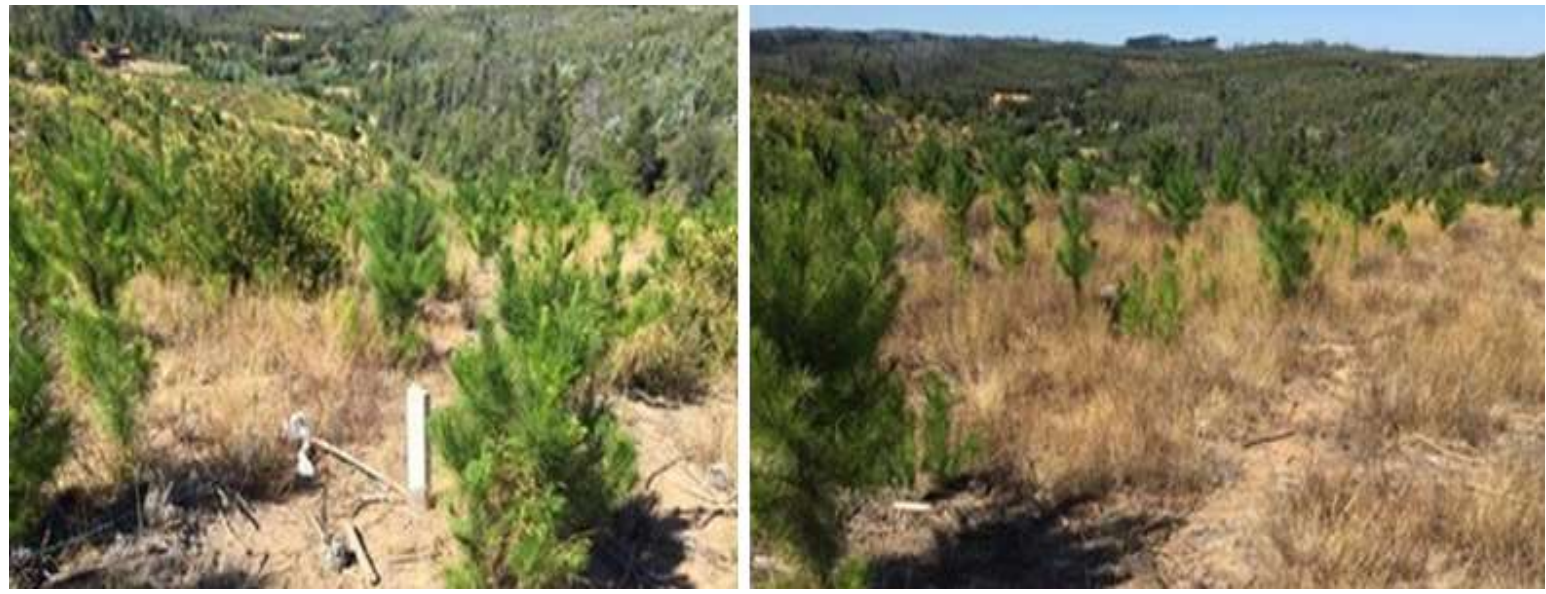

Figura 15. Unidad Lo Carmen, Vista General de la Parcela de Evaluación y del Rodal Manejado

\section{- Evaluación Unidad Santa Elisa}

Los resultados de la evaluación de la unidad Santa Elisa se presentan en el cuadro 11.

Cuadro 11. Situación de Plantas de Regeneración de Pino Radiata Manejadas en Unidad Santa Elisa

\begin{tabular}{|c|c|c|c|c|c|c|}
\hline \multirow{3}{*}{ Año } & \multicolumn{6}{|c|}{ Tratamiento de manejo } \\
\hline & \multicolumn{2}{|c|}{ Químico (ladera) } & \multicolumn{2}{|c|}{ Desbrozadora (plano) } & \multicolumn{2}{|c|}{ Desbrozadora (ladera) } \\
\hline & $\begin{array}{c}\mathrm{DC} \\
(\mathrm{mm})\end{array}$ & $\begin{array}{c}\text { Altura } \\
\text { (cm) }\end{array}$ & $\begin{array}{c}\mathrm{DC} \\
(\mathrm{mm})\end{array}$ & $\begin{array}{l}\text { Altura } \\
\text { (cm) }\end{array}$ & $\begin{array}{c}\mathrm{DC} \\
(\mathrm{mm})\end{array}$ & $\begin{array}{l}\text { Altura } \\
\text { (cm) }\end{array}$ \\
\hline 2019 & 18,0 & 102,4 & 18,3 & 93,5 & 12,4 & 80,2 \\
\hline 2020 & 34,5 & 199,1 & 29,1 & 154,0 & 20,0 & 130,8 \\
\hline
\end{tabular}



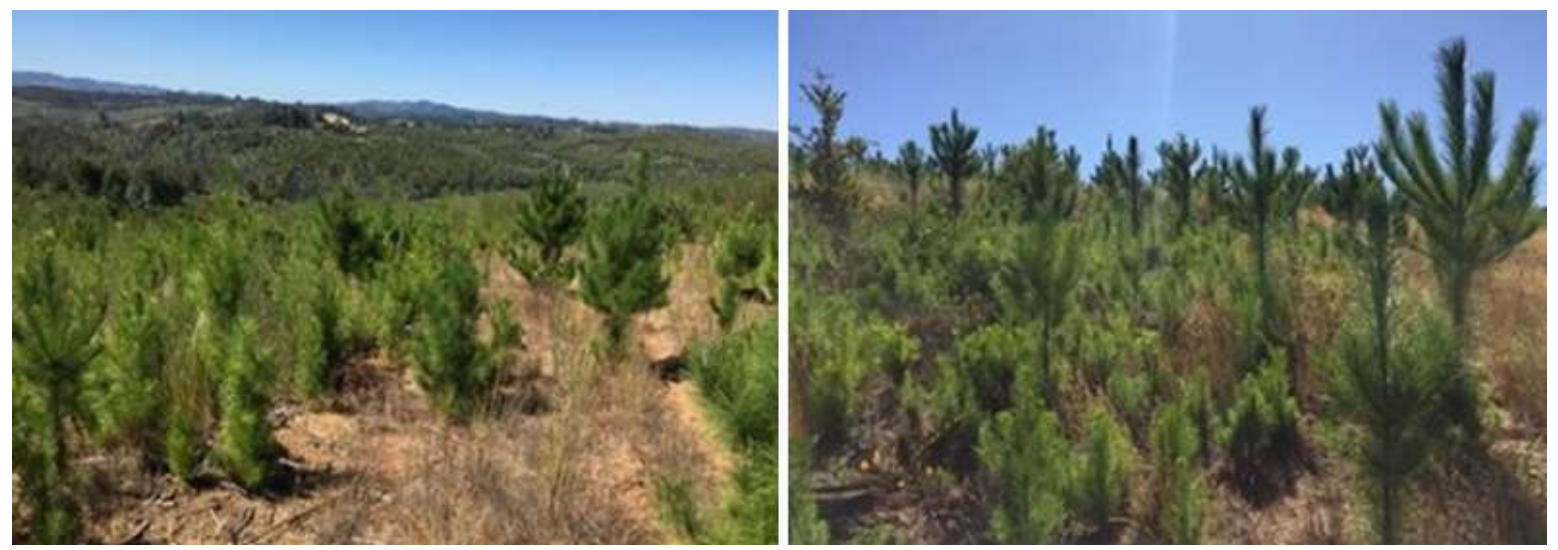

Figura 16. Unidad Santa Elisa, vista general del rodal manejado

Los resultados obtenidos derivan de las características propias de la especie, en cuanto a su estrategia de crecimiento, adaptación y propagación luego de un incendio forestal.

A partir de la información recopilada desde las unidades, es posible establecer que el control manual presentó los menores valores en crecimiento del diámetro de cuello en relación a los otros tipos de manejo utilizados.

La utilización del control químico utilizado genera los mayores valores en diámetro de cuello, al menos hasta dos años después de la intervención, que fue el periodo evaluado. Este mismo efecto se observa al considerar el crecimiento en altura.

La utilización de desbrozadora presenta variaciones en los resultados, ya que en una de las unidades su efecto fue similar e incluso inferior al obtenido con manejo manual.

Debe considerarse que los resultados son preliminares y de carácter referencial u orientativo, pues no responden a un diseño experimental que les otorgue validez estadística. Por lo mismo, se requiere de nuevas evaluaciones para detectar los reales efectos de este tipo de manejo y de su incidencia en la productividad del rodal.

Una vez definido cuál o cuáles serían los manejos más productivos, será necesario establecer nuevas unidades experimentales en donde se puedan comparar el crecimiento de rodales de pino radiata originados a través del manejo de la regeneración post cosecha o post incendios forestales, aunque la regeneración será más abundante en el segundo caso, y los resultados de una plantación tradicional establecida en el mismo tipo de sitio. En ese escenario se podrán evaluar parámetros de crecimiento y de productividad de los tipos de situaciones.

De obtenerse resultados que demuestren la validez de este tipo de manejo se podrían generar nuevos modelos a aplicar por pequeños y medianos propietarios forestales, con la consiguiente necesidad de programas de difusión, transferencia y, especialmente, de capacitación.

Ello implica también generar los antecedentes necesarios para favorecer su aplicación a través de acciones de fomento o incentivos específicos para este fin.

\section{CONCLUSIONES}

La densidad inicial y el tamaño de las plantas de la regeneración de pino radiata influyen sobre la eficiencia del manejo y consecuentemente en los resultados de los métodos aplicados.

No obstante, se debe considerar un breve período de crecimiento antes del manejo, que permita la expresión de las plantas regeneradas y con ello lograr una selección adecuada de ellas. 
De acuerdo con los primeros años de evaluación, el control manual presentó los menores valores en crecimiento del diámetro de cuello, por el contrario, el control químico registra los mayores valores en dicho parámetro, así como también en altura.

Se requiere de evaluaciones permanentes, de mediano y largo plazo, que permitan determinar la evolución de los efectos del tipo de manejo a medida que crecen los árboles, y de su incidencia en la productividad del rodal al final de la rotación.

Hasta el momento, los resultados confirman la potencialidad del manejo de la regeneración natural post incendio de pino radiata como herramientas de gestión forestal, para mejora la repoblación de áreas quemadas.

\section{REFERENCIAS}

Buesa V.A. (2003). Regeneración de pino radiata después de un incendio en el monte de UP Núm. 147 "Posadero". Montes. Revista de Ámbito Forestal 3(73): 46-48.

Castelán Lorenzo, M. \& Arteaga Martínez, B. (2009). Establecimiento de regeneración de Pinus patula Schl. et Cham., en cortas bajo el método de árboles padres. Revista Chapingo Serie Ciencias Forestales y del Ambiente 15(1): 49-57, 2009.

INFOR. (2018). Plantaciones afectadas por megaincendio según intensidad del fuego año 2017. Reporte de trabajo interno. Área Inventarios Forestales.

Pinilla, J.C., Luengo, K. \& Navarrete, M. (2019). Uso de la regeneración natural de pino radiata para la generación de plantaciones productivas como respuesta a la situación post incendios forestales. Informe MINAGRI.

Raga, Fernando; Valdebenito, Gerardo y Barros, Santiago. 2018. Reforestación de plantaciones forestales quemadas análisis de la viabilidad de aplicación de incentivos estatales y de la rentabilidad para el estado y particulares. Ciencia \& Investigación Forestal, 24(2): 87-100. https://doi.org/10.52904/0718-4646.2018.499. 